\title{
ON HARMONIC DISTURBANCE REJECTION OF AN UNDAMPED EULER-BERNOULLI BEAM WITH RIGID TIP BODY*
}

\author{
BaO-Zhu GuO ${ }^{1,2}$ AND QIONG ZHANG ${ }^{1}$
}

\begin{abstract}
A hybrid flexible beam equation with harmonic disturbance at the end where a rigid tip body is attached is considered. A simple motor torque feedback control is designed for which only the measured time-dependent angle of rotation and its velocity are utilized. It is shown that this control can impel the amplitude of the attached rigid tip body tending to zero as time goes to infinity.
\end{abstract}

Mathematics Subject Classification. 47F, 35K.

Received July 22, 2003. Revised November 14, 2003.

\section{INTRODUCTION}

In this paper, we consider a flexible beam rotated by a motor in a horizontal plane at one end and a tip body rigidly attached at the free end (see Fig. 1 below). This model fits a large class of real applications such as links of robot systems and space-shuttle arms in which high speed manipulation and long and slender geometrical dimensions are the major factors causing mechanical vibration. To achieve simultaneously the high speed, precise end point position and robustness of the flexible beam, the boundary control is one of the major strategies in production and space applications.

Let $\ell$ be the length of the beam, $\rho$ the uniform mass density per unit length, $E I$ the uniform flexural rigidity, $\tilde{M}$ the mass of the tip body attached, $\tilde{I}_{m}$ the moment of inertia of the motor and $\tilde{J}$ the moment of inertia associated with the tip body. Let $\theta(t)$ be the angle of rotation of motor at time $t$ and $w(x, t)$ be the deflection at position $x$ along moving axis and time $t$. Suppose that a) the deflection $w(x, t)$ is small and any extension is neglected; b) all terms $\dot{\theta}^{2}$ are negligible; c) the twist of the beam is ignored; d) both the distance between the beam's tip point and the intersection of the beam's tip tangent with a perpendicular plane passing through the tip body's center and the damping effects are ignored. Then $w(x, t)$ satisfies the following Euler-Bernoulli beam equation and the Newton-Euler rigid-body equations [12]:

$$
\left\{\begin{array}{l}
\rho w_{t t}(x, t)+E I w_{x x x x}(x, t)=-x \ddot{\theta}(t), 0<x<\ell, t>0, \\
w(0, t)=w_{x}(0, t)=0 \\
E I w_{x x x}(\ell, t)=\tilde{M}\left[\ell \ddot{\theta}(t)+w_{t t}(\ell, t)\right], \\
-E I w_{x x}(\ell, t)=\tilde{J}\left[\ddot{\theta}(t)+w_{x t t}(\ell, t)\right] .
\end{array}\right.
$$

Keywords and phrases. Beam equation, disturbance rejection, noncollocated control, strong stability.

* Supported by the National Natural Science Foundation of China.

1 Academy of Mathematics and System Sciences, Academia Sinica, Beijing 100080, China; e-mail: bzguo@iss03.iss.ac.cn

2 Departement of Computational and Applied Mathematics, University of the Witwatersrand, Private Bag-3, Wits-2050, Johannesburg, South Africa.

(C) EDP Sciences, SMAI 2004 


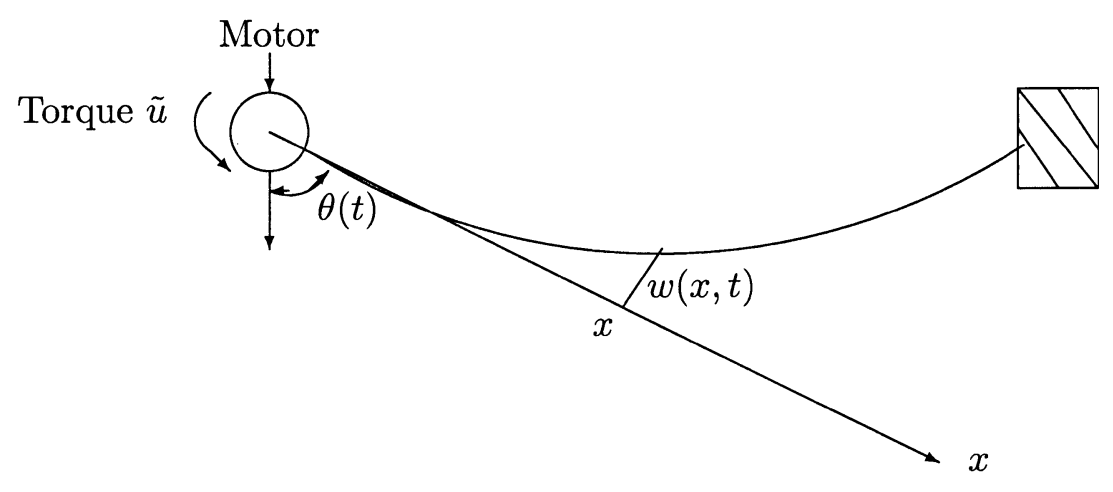

FIgURE 1. The vibration control of a flexible link with a rigid tip body.

The equation of motion of rotation motor is [12]

$$
\tilde{I}_{m} \ddot{\theta}(t)=\tilde{u}(t)+E I w_{x x}(0, t)
$$

where $\tilde{u}(t)$ is the torque developed by the motor and applied at the root of the beam. If we define $\tilde{y}(x, t)=$ $w(x, t)+x \theta(t)$ as the total deflection of the beam [1]. Then $\tilde{y}$ satisfies $[4,5,15]$

$$
\left\{\begin{array}{l}
\rho \tilde{y}_{t t}(x, t)+E I \tilde{y}_{x x x x}(x, t)=0,0<x<\ell, t>0 \\
\tilde{y}(0, t)=0 \\
E I \tilde{y}_{x x}(0, t)-\tilde{I}_{m} \tilde{y}_{x t t}(0, t)+\tilde{u}(t)=0 \\
E I \tilde{y}_{x x x}(\ell, t)-\tilde{M} \tilde{y}_{t t}(\ell, t)=0 \\
E I \tilde{y}_{x x}(\ell, t)+\tilde{J} \tilde{y}_{x t t}(\ell, t)=0
\end{array}\right.
$$

The model (1.3) was established again in [15] based on [7]. The stability by using the feedback of measured time-dependent angular and its velocity at $x=0$ was studied in $[4,5]$. Most existing boundary control design methods for flexible system are using the collocated actuators and sensors based on passive principle because the collocated system with rate feedback is inherently stable (see e.g. [2,9], etc.). However, as it was indicated in [11] that with such collocated measurements, the vibration of the system are not controlled well enough. On the other hand, some effort has been made to design the noncollocated control for the flexible systems, for instance [3] and [13] where some alternative noncollocated measurements are proposed to deal with the difficulty of presence of the right half plane zeros in the transfer function and the finite dimensional compensator was used to stabilize the damped flexible systems. In this paper, we consider a different problem: disturbance rejection through noncollocated control by which we mean that the control is at the one end and the output of concern is at the another.

For notational simplicity, we make the following transformation

$$
y(x, t)=\tilde{y}\left(\ell x, \sqrt{\frac{\rho \ell^{4}}{E I}} t\right), u(t)=\frac{\ell^{2}}{E I} \tilde{u}\left(\sqrt{\frac{\rho \ell^{4}}{E I}} t\right), I_{m}=\frac{\tilde{I}_{m}}{\ell^{3} \rho}, M=\frac{\tilde{M}}{\ell \rho}, J=\frac{\tilde{J}}{\ell^{3} \rho}
$$

and consider the harmonic disturbance at the end where the rigid tip bogy is attached. After that $y$ satisfies

$$
\left\{\begin{array}{l}
y_{t t}(x, t)+y_{x x x x}(x, t)=0,0<x<1, t>0, \\
y(0, t)=0, \\
I_{m} y_{x t t}(0, t)-y_{x x}(0, t)=u(t), \\
J y_{x t t}(1, t)+y_{x x}(1, t)=\rho_{J} \sin \left(\omega_{J} t+\psi_{J}\right), \\
M y_{t t}(1, t)-y_{x x x}(1, t)=\rho_{M} \sin \left(\omega_{M} t+\psi_{M}\right)
\end{array}\right.
$$




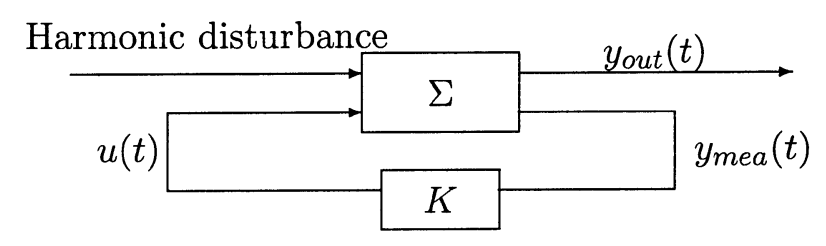

FiguRE 2. The block of disturbance rejection by the measured feedback.

where in the input harmonic disturbances $\rho_{J} \sin \left(\omega_{J} t+\psi_{J}\right)$ and $\rho_{M} \sin \left(\omega_{M} t+\psi_{M}\right)$, the frequencies $\omega_{J}$ and $\omega_{M}$ are assumed to be known but both the amplitudes $\rho_{J}, \rho_{M}$ and the phases $\psi_{J}, \psi_{M}$ are uncertain constants. Suppose that

$$
y_{\text {out }}(t)=y(1, t), \forall t \geq 0
$$

is the output to be concerned and the measured output is given by

$$
y_{\text {mea }}(t)=\left(y_{x}(0, t), y_{x t}(0, t)\right), \forall t \geq 0
$$

The objective of this paper is to design the control input $u(t)$ by using only the measured output so that

$$
\lim _{t \rightarrow \infty} y_{\text {out }}(t)=\lim _{t \rightarrow \infty} y(1, t)=0
$$

Disturbance rejection or attenuation is one of the most important and widely studied areas in control theory. One of the main principles is the internal model principle, that is, making the transfer function of controller have poles at the frequencies of the disturbances. We refer this approach to [6] and the references therein where the transfer function is required to be in Callier-Desoer algebra. The results of [6] were extended in [10] that for the well-posed system [14] with disturbance, the output $y_{\text {out }} \in L_{\gamma}^{2}(0, \infty)$ for some $\gamma>0$. The result of [10] is then applied to the rejection of external noise in the structural acoustics model and two coupled beams. Very different to internal model principle, in this paper, we propose the following simple controller by using only measured output:

$$
\left\{\begin{array}{l}
\xi_{1}^{\prime}(t)=\omega \xi_{2}(t) \\
\xi_{2}^{\prime}(t)=-\omega \xi_{1}(t)-k \xi_{2}(t)+k y_{t x}(0, t), \\
u(t)=-\alpha y_{x}(0, t)+k \xi_{2}-k y_{t x}(0, t)
\end{array}\right.
$$

where the nonzero constants $\alpha, k$ and $\omega$ can be turned in practical design. We will show that controller (1.8) can stabilize not only the system (1.4) without disturbance but also impel the output concerned tending to standstill in the presence of disturbances as in the equation (1.4). The process can be illustrated by a typical block in $H_{\infty}$ control framework as in Figure 2 above.

\section{WELL-POSEDNESS AND STABILITY WITHOUT DISTURBANCE}

Suppose there are no disturbances at the rigid tip body attached end. Then the closed-loop system of (1.4) with control law (1.8) becomes

$$
\left\{\begin{array}{l}
y_{t t}(x, t)+y_{x x x x}(x, t)=0,0<x<1, t \geq 0 \\
y(0, t)=0 \\
I_{m} y_{t t x}(0, t)-y_{x x}(0, t)+\alpha y_{x}(0, t)=k \xi_{2}-k y_{t x}(0, t) \\
J y_{t t x}(1, t)+y_{x x}(1, t)=0 \\
M y_{t t}(1, t)-y_{x x x}(1, t)=0 \\
\xi_{1}^{\prime}(t)=\omega \xi_{2}(t) \\
\xi_{2}^{\prime}(t)=-\omega \xi_{1}(t)-k \xi_{2}(t)+k y_{t x}(0, t)
\end{array}\right.
$$


Consider (2.1) in the state Hilbert space $H=V \times L^{2}(0,1) \times \mathbb{C}^{5}, V=\left\{z \in H^{2}(0,1) \mid z(0)=0\right\}$ endowed with the inner product norm

$$
\begin{aligned}
\left\|\left(y, w, v_{0}, v_{1}, v_{2}, \xi_{1}, \xi_{2}\right)\right\|_{H}^{2}= & \int_{0}^{1}\left|y_{x x}(x)\right|^{2} \mathrm{~d} x+\alpha\left|y_{x}(0)\right|^{2}+\int_{0}^{1}|w(x)|^{2} \mathrm{~d} x \\
& +I_{m}\left|v_{0}\right|^{2}+J\left|v_{1}\right|^{2}+M\left|v_{2}\right|^{2}+\left|\xi_{1}\right|^{2}+\left|\xi_{2}\right|^{2} .
\end{aligned}
$$

Then the system (2.1) can be written as an evolution equation in $H$

$$
\dot{Y}(t)=A Y(t)
$$

where $Y(t)=\left(y(\cdot, t), y_{t}(\cdot, t), y_{t x}(0, t), y_{t x}(1, t), y_{t}(1, t), \xi_{1}, \xi_{2}\right)$ is the state and the system operator $A:(H \supset)$ $D(A) \rightarrow H$ is defined as follows:

$$
\begin{gathered}
A\left(\begin{array}{c}
y \\
w \\
v_{0} \\
v_{1} \\
v_{2} \\
\xi_{1} \\
\xi_{2}
\end{array}\right)=\left(\begin{array}{c}
w \\
-y_{x x x x} \\
\frac{1}{I_{m}}\left(y_{x x}(0)-\alpha y_{x}(0)+k \xi_{2}-k w_{x}(0)\right) \\
-\frac{1}{J} y_{x x}(1) \\
\frac{1}{M} y_{x x x}(1) \\
\omega \xi_{2} \\
-\omega \xi_{1}-k \xi_{2}+k w_{x}(0)
\end{array}\right), \\
D(A)=\left\{\left(y, w, v_{0}, v_{1}, v_{2}, \xi_{1}, \xi_{2}\right) \in H \mid \begin{array}{l}
y \in H^{4}(0,1), w \in V, v_{0}=w_{x}(0), \\
v_{1}=w_{x}(1), v_{2}=w(1)
\end{array}\right\} .
\end{gathered}
$$

Theorem 2.1. Let $A$ be given by (2.3). Then A generates a $C_{0}$-semigroup $T(t)$ of contractions on $H$. Moreover, $0 \in \rho(A)$ and $A^{-1}$ is compact. Therefore, $A$ is discrete and $\sigma(A)$ only consists of isolated eigenvalues.

Proof. For any $Y=\left(y, w, v_{0}, v_{1}, v_{2}, \xi_{1}, \xi_{2}\right) \in D(A)$, it has

$$
\begin{aligned}
\operatorname{Re}\langle A Y, Y\rangle_{H}= & \operatorname{Re}\left[\int_{0}^{1} w_{x x} \overline{y_{x x}} \mathrm{~d} x+\alpha w_{x}(0) \overline{y_{x}(0)}-\int_{0}^{1} y_{x x x x} \bar{w} \mathrm{~d} x\right. \\
\left(y_{x x}(0)-\alpha y_{x}(0)+k \xi_{2}-k w_{x}(0) \overline{w_{x}(0)}-y_{x x}(1) \overline{w_{x}(1)}\right. & \left.\quad+y_{x x x}(1) \overline{w(1)}+\omega \xi_{2} \overline{\xi_{1}}+\left(-\omega \xi_{1}-k \xi_{2}+k w_{x}(0)\right) \overline{\xi_{2}}\right] \\
= & -k\left|w_{x}(0)-\xi_{2}\right|^{2} \leq 0 .
\end{aligned}
$$

Hence $A$ is dissipative. Furthermore, for any fixed but arbitrary element $f=\left(f_{1}, f_{2}, f_{3}, f_{4}, f_{5}, f_{6}, f_{7}\right)$ in $H$, solving equation

$$
A\left(\begin{array}{c}
y \\
w \\
v_{0} \\
v_{1} \\
v_{2} \\
\xi_{1} \\
\xi_{2}
\end{array}\right)=\left(\begin{array}{c}
w \\
-y_{x x x x} \\
\frac{1}{I_{m}}\left(y_{x x}(0)-\alpha y_{x}(0)+k \xi_{2}-k w_{x}(0)\right) \\
-\frac{1}{J} y_{x x}(1) \\
\frac{1}{M} y_{x x x}(1) \\
\omega \xi_{2} \\
-\omega \xi_{1}-k \xi_{2}+k w_{x}(0)
\end{array}\right)=\left(\begin{array}{c}
f_{1} \\
f_{2} \\
f_{3} \\
f_{4} \\
f_{5} \\
f_{6} \\
f_{7}
\end{array}\right)
$$


gives

and

$$
\begin{aligned}
y(x)= & -\int_{0}^{x} \int_{0}^{s_{1}} \int_{s_{2}}^{1} \int_{s_{3}}^{1} f_{2}(s) \mathrm{d} s \mathrm{~d} s_{3} \mathrm{~d} s_{2} \mathrm{~d} s_{1}-\frac{1}{2} J x^{2} f_{4}-M\left(\frac{1}{2} x^{2}-\frac{1}{6} x^{3}\right) f_{5} \\
& +\frac{1}{\alpha} x\left(-J f_{4}-M f_{5}+\int_{0}^{1} \int_{s_{1}}^{1} f_{2}(s) \mathrm{d} s \mathrm{~d} s_{1}+\frac{k}{\omega} f_{6}-k f_{1 x}(0)-I_{m} f_{3}\right)
\end{aligned}
$$

$$
\begin{aligned}
& w=f_{1}, v_{0}=f_{1 x}(0), v_{1}=f_{1 x}(1), v_{2}=f_{1}(1), \\
& \xi_{2}=\frac{1}{\omega} f_{6}, \xi_{1}=\frac{k}{\omega} f_{1 x}(0)-\frac{k}{\omega^{2}} f_{6}-\frac{1}{\omega} f_{7}
\end{aligned}
$$

and the compactness follows from the Sobolev's embedding theorem applied to $A^{-1}$.

When the control is assigned to be $u(t)=-\alpha y_{x}(0, t)$ only, that is to say $\xi_{2}=y_{t x}(0, t)$ in equation $(2.1)$, we obtain a conservative system associated which the system operator, denoted by $A_{0}$ with $D\left(A_{0}\right)=D(A)$, is also discrete and moreover, skew-adjoint in $H$ :

$$
A_{0}\left(\begin{array}{c}
y \\
w \\
v_{0} \\
v_{1} \\
v_{2} \\
\xi_{1} \\
\xi_{2}
\end{array}\right)=\left(\begin{array}{c}
w \\
-y_{x x x x} \\
\frac{1}{I_{m}}\left(y_{x x}(0)-\alpha y_{x}(0)\right) \\
-\frac{1}{J} y_{x x}(1) \\
\frac{1}{M} y_{x x x}(1) \\
\omega \xi_{2} \\
-\omega \xi_{1}
\end{array}\right)
$$

It follows from a well-known fact in functional analysis that all eigenvalues of $A_{0}$ lie in the imaginary axis which are symmetric with respect to the real axis. So, we may assume $\sigma\left(A_{0}\right)=\left\{\left(i \tau^{2},-i \tau^{2}\right) \mid \tau\right.$ are positive $\}$. A direct computation shows that $\tau$ satisfies the following characteristic equation:

$$
\begin{aligned}
& \left(-I_{m} \tau^{4}+\alpha\right)\left[1+M J \tau^{4}+\left(M \tau-J \tau^{3}\right) \sinh \tau \cos \tau\right. \\
& \left.-\left(M \tau+J \tau^{3}\right) \cosh \tau \sin \tau+\left(1-M J \tau^{4}\right) \cosh \tau \cos \tau\right] \\
& +\tau\left[-2 J \tau^{3} \cosh \tau \cos \tau+\left(-1+M J \tau^{4}\right) \cosh \tau \sin \tau\right. \\
& \left.+\left(1-M J \tau^{4}\right) \sinh \tau \cos \tau-2 M \tau \sinh \tau \sin \tau\right]=0
\end{aligned}
$$

Since $\tau$ is positive, a close examination of characteristic equation (2.4) reveals that

$$
\cos \tau=-\frac{\sin \tau}{M \tau}+O\left(\tau^{-2}\right)
$$

whose solution can asymptotically found to be [5]

$$
\tau=\tau_{n}=\left(n-\frac{1}{2}\right) \pi+O\left(n^{-1}\right) \text { for sufficiently large } n .
$$

As for those zeros of (2.4) closed to the origin one can easily find them through numerical methods.

Theorem 2.2. Choose $\tau=\sqrt{|\omega|}$ not to be the root of (2.4). Then the semigroup $T(t)$ generated by $A$ is strongly stable.

Proof. Since $T(t)$ is bounded and $A$ is discrete, by a well-known sufficient characterization condition on strong stability of bounded $C_{0}$-semigroup (see e.g. Th. 3.26 of [8]), the proof will be accomplished by showing that there is no eigenvalue of $A$ on the imaginary axis. Suppose now that $0 \neq \lambda \in \mathbb{R}$. Solving eigenvalue problem of the following

$$
A Y=i \lambda Y, \forall Y=\left(y, w, v_{0}, v_{1}, v_{2}, \xi_{1}, \xi_{2}\right) \in D(A),
$$


we find that $y, \xi_{1}$ and $\xi_{2}$ satisfy

$$
\left\{\begin{array}{l}
-\lambda^{2} y+y_{x x x x}=0 \\
y(0)=0 \\
-\lambda^{2} I_{m} y_{x}(0)-y_{x x}(0)+\alpha y_{x}(0)-k \xi_{2}+i \lambda k y_{x}(0)=0 \\
-\lambda^{2} J y_{x}(1)+y_{x x}(1)=0 \\
-\lambda^{2} M y(1)-y_{x x x}(1)=0 \\
i \lambda \xi_{1}-\omega \xi_{2}=0 \\
i \lambda \xi_{2}+\omega \xi_{1}+k \xi_{2}-k i \lambda y_{x}(0)=0
\end{array}\right.
$$

In light of the fact that $0=\operatorname{Re}\langle A Y, Y\rangle_{H}=-k\left|i \lambda y_{x}(0)-\xi_{2}\right|^{2}$ as in the proof of Theorem 2.1, we obtain that $i \lambda y_{x}(0)=\xi_{2}$. Hence $(2.7)$ becomes

$$
\left\{\begin{array}{l}
-\lambda^{2} y+y_{x x x x}=0 \\
y(0)=0 \\
-\lambda^{2} I_{m} y_{x}(0)-y_{x x}(0)+\alpha y_{x}(0)=0 \\
-\lambda^{2} J y_{x}(1)+y_{x x}(1)=0 \\
-\lambda^{2} M y(1)-y_{x x x}(1)=0 \\
i \lambda \xi_{1}-\omega \xi_{2}=0 \\
i \lambda \xi_{2}+\omega \xi_{1}=0
\end{array}\right.
$$

It is clear that if one of $\xi_{1}$ and $\xi_{2}$ is not identically zero, then the last two equalities of (2.8) imply that $\lambda=0$. Suppose first that both $\xi_{1}$ and $\xi_{2}$ are not identically zero. Then $\lambda^{2}=-\omega^{2}$ and since the first five equalities of (2.8) are nothing but the eigen equation of $A_{0}$, it follows that $\sqrt{|\omega|}$ is a root of (2.4). This contradicts the hypothesis. While both $\xi_{1}$ and $\xi_{2}$ are identically zero, then $y_{x}(0)=0$ from the proved fact that $i \lambda y_{x}(0)=\xi_{2}$. In this case, (2.8) becomes

$$
\left\{\begin{array}{l}
-\lambda^{2} y+y_{x x x x}=0 \\
y(0)=y_{x}(0)=y_{x x}(0)=0 \\
-\lambda^{2} J y_{x}(1)+y_{x x}(1)=0 \\
-\lambda^{2} M y(1)-y_{x x x}(1)=0
\end{array}\right.
$$

Put $\lambda=\tau^{2}, \tau>0$. Then the solution to the equation above takes the form $y(x)=\sinh \tau x-\sin \tau x$ with $\tau$ satisfying

$$
\begin{aligned}
& -\tau^{3} J(\cosh \tau-\cos \tau)+(\sinh \tau+\sin \tau)=0 \\
& -\tau M(\sinh \tau-\sin \tau)-(\cosh \tau+\cos \tau)=0
\end{aligned}
$$

from which we can readily obtain

$$
-\tau^{2} J\left(\cosh ^{2} \tau-\cos ^{2} \tau\right)=M\left(\sinh ^{2} \tau+\sin ^{2} \tau\right)
$$

and hence $\lambda$ must be identically zero. We have once again had a contradiction. The result follows.

Remark 2.1. Theorem 2.2 is the best stability result we can hope by the assigned output feedback for the exponentially stability needs additional measured signals, for which we refer to [5] for more details.

\section{Disturbance ReJeCtion}

In this section, we turn to our main object of disturbance rejection. Obviously, the condition of Theorem 2.2 is necessary for this purpose. We will discuss two different cases: $\rho_{J}=0$ and $\rho_{M}=0$. First we investigate the first case of $\rho_{J}=0$. 
Theorem 3.1. Suppose $\rho_{J}=0$ and $\omega=\omega_{M}$. If $\tau=\sqrt{\left|\omega_{M}\right|}$ is not the root of (2.4) but one of the following equation

$$
\begin{aligned}
& {\left[2 \tau \sinh \tau+\left(\alpha-I_{m} \tau^{4}\right)(\cosh \tau-\cos \tau)\right]\left[-2 J \tau^{4} \cos \tau-2 \tau \sin \tau\right.} \\
& \left.+\left(\alpha-I_{m} \tau^{4}\right)\left(-J \tau^{3} \sinh \tau+\cosh \tau-J \tau^{3} \sin \tau+\cos \tau\right)\right] \\
& -\left[2 \tau \sin \tau+\left(\alpha-I_{m} \tau^{4}\right)(\cosh \tau-\cos \tau)\right]\left[-2 J \tau^{4} \cosh \tau+2 \tau \sinh \tau\right. \\
& \left.+\left(\alpha-I_{m} \tau^{4}\right)\left(-J \tau^{3} \sinh \tau+\cosh \tau-J \tau^{3} \sin \tau+\cos \tau\right)\right]=0
\end{aligned}
$$

by properly choosing the parameter $\alpha$, then the output of the system (1.4) and (1.8) satisfies $\lim _{t \rightarrow \infty} y_{\text {out }}(t)=$ $\lim _{t \rightarrow \infty} y(1, t)=0$.

Remark 3.1. Similar to that of (2.4), the positive solutions of (3.1) are of the following asymptotic expansion:

$$
\cos \tau=\left(\frac{1}{I_{m}}+\frac{1}{J}\right) \frac{\sin \tau}{\tau^{3}}+\mathcal{O}\left(\tau^{-4}\right)
$$

and hence

$$
\tau=\tau_{n}=\left(n-\frac{1}{2}\right) \pi+\mathcal{O}\left(n^{-3}\right) \text { for } n \text { sufficiently large. }
$$

Proof of Theorem 3.1. We begin by constructing a particular solution $\tilde{y}(x, t)=\phi(x) \sin \left(\omega_{M} t+\psi_{M}\right)$ for the following equation:

$$
\left\{\begin{array}{l}
\tilde{y}_{t t}(x, t)+\tilde{y}_{x x x x}(x, t)=0,0<x<1, t \geq 0, \\
\tilde{y}(0, t)=0 \\
I_{m} \tilde{y}_{t t x}(0, t)-\tilde{y}_{x x}(0, t)+\alpha \tilde{y}_{x}(0, t)=0, \\
J \tilde{y}_{t t x}(1, t)+\tilde{y}_{x x}(1, t)=0, \\
\tilde{y}(1, t)=0 .
\end{array}\right.
$$

This is possible whenever $\phi$ satisfies

$$
\left\{\begin{array}{l}
\phi^{(4)}(x)-\omega_{M}^{2} \phi(x)=0,0<x<1 \\
\phi(0)=0 \\
\phi^{\prime \prime}(0)=\left(\alpha-I_{m} \omega_{M}^{2}\right) \phi^{\prime}(0) \\
\phi^{\prime \prime}(1)=J \omega_{M}^{2} \phi^{\prime}(1) \\
\phi(1)=0
\end{array}\right.
$$

Solving equation (3.4) shows that all is well-there does exist a nonzero solution to (3.4) under the hypothesis, which is justified by observing that (3.1) is nothing but the characteristic equation which guarantees the existence of a nonzero solution to the equation (3.4). Finding this solution is a direct computation, and since it poses no special difficulty, we just write down it explicitly:

$$
\begin{aligned}
\phi(x) & =-\left[2 \tau \sin \tau+\left(\alpha-I_{m} \tau^{4}\right)(\cosh \tau-\cos \tau)\right] \sinh \tau x \\
& +\left[2 \tau \sinh \tau+\left(\alpha-I_{m} \tau^{4}\right)(\cosh \tau-\cos \tau)\right] \sin \tau x \\
& +\left(\alpha-I_{m} \tau^{4}\right)(\sinh \tau-\sin \tau)(\cosh \tau x-\cos \tau x) .
\end{aligned}
$$

Therefore,

$$
\begin{aligned}
\phi^{\prime \prime \prime}(1) & =\tau^{3}\left[-2 \tau \sin \tau \cosh \tau-2 \tau \sinh \tau \cos \tau-2\left(\alpha-I_{m} \tau^{4}\right) \sinh \tau \sin \tau\right] \\
& =2 \tau^{7} \mathrm{e}^{-\tau}\left[-\tau^{-3} \cos \tau+\left(I_{m}-\tau^{-3}-\alpha \tau^{-4}\right) \sin \tau+\mathcal{O}\left(\mathrm{e}^{-\tau}\right)\right]
\end{aligned}
$$

which is not identically zero whenever $\tau$ large enough by comparing with (3.2). 
Now that define

$$
\left\{\begin{array}{l}
y_{d}(x, t)=-\frac{\rho_{M} \phi(x) \sin \left(\omega_{M} t+\psi_{M}\right)}{\phi^{\prime \prime \prime}(1)} \\
\xi_{1 d}(t)=-\frac{\rho_{M} \omega_{M} \phi^{\prime}(0) \sin \left(\omega_{M} t+\psi_{M}\right)}{\phi^{\prime \prime \prime}(1)} \\
\xi_{2 d}(t)=-\frac{\rho_{M} \omega_{M} \phi^{\prime}(0) \cos \left(\omega_{M} t+\psi_{M}\right)}{\phi^{\prime \prime \prime}(1)}
\end{array}\right.
$$

Then a direct computation shows that $\left(y, \xi_{1}, \xi_{2}\right)=\left(y_{d}, \xi_{1 d}, \xi_{2 d}\right)$ given by (3.7) satisfy (1.4) and (1.8) as $\rho_{J}=0$ and the most importantly,

$$
y_{d}(1, t)=0
$$

Decompose the solution of (1.4) and (1.8) as

$$
y(x, t)=y_{s}(x, t)+y_{d}(x, t), \xi_{1}=\xi_{1 s}+\xi_{1 d}, \xi_{2}=\xi_{2 s}+\xi_{2 d}
$$

It is obviously that $\left(y, \xi_{1}, \xi_{2}\right)=\left(y_{s}, \xi_{1 s}, \xi_{2 s}\right)$ is a solution of $(2.1)$ which is strongly stable by virtue of Theorem 2.2 , in particular

$$
\lim _{t \rightarrow \infty} y_{s}(1, t)=0
$$

This together with (3.8) finally verifies that

$$
\lim _{t \rightarrow \infty} y(1, t)=0
$$

Along the same line of proof of Theorem 3.1, we have the disturbance rejection result for the case of $\rho_{M}=0$.

Theorem 3.2. Suppose $\rho_{M}=0$ and $\omega=\omega_{J}$. If $\tau=\sqrt{\left|\omega_{J}\right|}$ is not the root of (2.4) but that of the following equation

$$
\begin{aligned}
& {\left[2 \tau \sinh \tau+\left(\alpha-I_{m} \tau^{4}\right)(\cosh \tau-\cos \tau)\right]\left[2 M \tau^{2} \sin \tau-2 \tau \cos \tau\right.} \\
& \left.+\left(\alpha-I_{m} \tau^{4}\right)(M \tau \cosh \tau+\sinh \tau-M \tau \cos \tau-\sin \tau)\right] \\
& -\left[2 \tau \sin \tau+\left(\alpha-I_{m} \tau^{4}\right)(\cosh \tau-\cos \tau)\right]\left[2 M \tau^{2} \sinh \tau+2 \tau \cosh \tau\right. \\
& \left.+\left(\alpha-I_{m} \tau^{4}\right)(M \tau \cosh \tau+\sinh \tau-M \tau \cos \tau-\sin \tau)\right]=0
\end{aligned}
$$

by properly choosing the parameter $\alpha$, then the output of the system (1.4) and (1.8) satisfies $\lim _{t \rightarrow \infty} y_{\text {out }}(t)=$ $\lim _{t \rightarrow \infty} y(1, t)=0$.

Remark 3.2. It should be pointed out that we use low order controller (1.8) to stabilize the displacement of the tip body. If we choose the output as $y_{\text {out }}(t)=\left(y(1, t), y_{x}(0, t)\right)$, we need design a high order controller of the following form so that additional constants $\beta$ or $m$ can be regulated to ensure the particular solution of (3.3) satisfying $\tilde{y}_{x}(1, t)=0$.

$$
\left\{\begin{array}{l}
\xi_{1}^{\prime}(t)=\omega \xi_{2}(t) \\
\xi_{2}^{\prime}(t)=-\omega \xi_{1}(t)-k \xi_{2}(t)+k y_{t x}(0, t) \\
\eta_{1}^{\prime}(t)=\eta_{2}(t) \\
\eta_{2}^{\prime}(t)=-(\alpha+\beta) / m \eta_{1}(t)+\alpha / m y(0, t) \\
u(t)=-\alpha y_{x}(0, t)+k \xi_{2}-k y_{t x}(0, t)+\alpha \eta_{1}(t)
\end{array}\right.
$$

Unfortunately, under this controller, the corresponding system operator $A$ of the closed-loop system is not dissipative and hence it is not easy to determine its asymptotic stability. The related work is still going on. 


\section{Concluding Remarks}

In this paper, we start directly from a partial differential equation model of beam equation and propose a simple integrator type controller where only measured angular of rotation of motor and its velocity at one end of the beam are utilized in the design of feedback control law to stabilize the output of concern in the presence of the harmonic disturbance at the another end where the rigid tip body is attached. The key ideas lie in a) the controller must stabilize the system without disturbance; b) constructing a particular solution with fixed end position through spectral analysis. The rigorous mathematical proof is presented for the PDE model without any damping.

\section{REFERENCES}

[1] R.H. Cannon, Jr. and E. Schmitz, Inital experiments on the end-point control of a flexible one-link Robot. Inter. J. Robotics Res. 3 (1984) 62-75.

[2] G. Chen, M.C. Delfour, A.M. Krall and G. Payre, Modeling, stabilization and control of serially connected beam. SIAM J. Control Optim. 25 (1987) 526-546.

[3] P.A. Chodavarapu and M.W. Spong, On noncolocted control of a single flexible link, in Proc. of the 1996 IEEE International Conference on Robotics and Automation, Minneapolis, Minnesota (April 1996) 1101-1106.

[4] B.Z. Guo and Q. Song, Tracking control of a flexible beam by nonlinear boundary feedback. J. Appl. Math. Stochastic Anal. 8 (1995) 47-58.

[5] B.Z. Guo, Riesz basis approach to the tracking control of a flexible beam with a tip rigid body without dissipativity. Optim. Methods Softw. 17 (2002) 655-681.

[6] T. Hämäläinen and S. Pohjolainen, A finite dimensional robust controller for systems in the CD-algebra. IEEE Trans. Autom. Control 45 (2000) 421-431.

[7] S. Lee and J.L. Junkins, Explicit generalization of Langrange's equations for hybrid coordinate dynamic systems, J. Guid. Control Dyn. 15 (1992) 1443-1452.

[8] Z.H. Luo, B.Z. Guo and Ö. Morgül, Stability and Stabilization of Linear Dimensional Systems with Applications. SpringerVerlag, London (1999).

[9] J.C. Oostveen and R.F. Curtain, Robustly stabilizing controllers for dissipative infinite-dimensional systems with collocated actuators and sensors. Automatica 36 (2000) 337-348.

[10] R. Rebarber and G. Weiss, Internal model based tracking and disturbance rejection for stable well-posed systems. Automatica, in press.

[11] Y. Sakawa, F. Matsuno and S. Fukushima, Modeling and feedback control of a flexible arm. J. Rob. Syst. 2 (1985) 453-472.

[12] Y. Sakawa and Z.H. Luo, Modeling and control of coupled bending and torsional vibrations of flexible beam. IEEE Trans. Autom. Control 34 (1989) 970-977.

[13] V.A. Spetor and H. Flashner, Modeling and design implification of noncollocated control in flexible systems. J. Dyn. Syst. Meas. Control 112 (1990) 186-193.

[14] G. Weiss and R. Rebarber, Optimizability and estimatability for infinite-dimensional linear systems. SIAM J. Control Optim. 39 (2001) 1204-1232.

[15] K. Yuan and L. Liu, Achieving mininmum phase transfer function for a noncollocated single-link flexible manipulator. Asian J. Control 2 (2000) 179-191. 